\title{
Quantum-state-preserving Frequency Conversion Using Four-wave Mixing
}

\author{
Andersen, Lasse Mejling; Reddy, Dileep V.; McKinstrie, Colin J.; Raymer, Michael G.; Rottwitt, Karsten
}

Published in:

Nonlinear Optics Technical Digest

Publication date:

2013

Document Version

Publisher's PDF, also known as Version of record

Link back to DTU Orbit

Citation (APA):

Andersen, L. M., Reddy, D. V., McKinstrie, C. J., Raymer, M. G., \& Rottwitt, K. (2013). Quantum-statepreserving Frequency Conversion Using Four-wave Mixing. In Nonlinear Optics Technical Digest (pp. NTu1A.2). Optical Society of America.

\section{General rights}

Copyright and moral rights for the publications made accessible in the public portal are retained by the authors and/or other copyright owners and it is a condition of accessing publications that users recognise and abide by the legal requirements associated with these rights.

- Users may download and print one copy of any publication from the public portal for the purpose of private study or research.

- You may not further distribute the material or use it for any profit-making activity or commercial gain

- You may freely distribute the URL identifying the publication in the public portal 


\title{
Quantum-state-preserving Frequency Conversion Using Four-wave Mixing
}

\author{
L. Mejling, ${ }^{1, *}$ D. V. Reddy, ${ }^{2}$ C. J. McKinstrie, ${ }^{3}$ M. G. Raymer, ${ }^{2}$ and K. Rottwitt ${ }^{1}$ \\ ${ }^{1}$ Department of Photonics Engineering, Technical University of Denmark, DK-2800 Kgs. Lyngby, Denmark \\ ${ }^{2}$ Department of Physics, University of Oregon, Eugene, Oregon 97403, USA \\ ${ }^{3}$ Bell Laboratories, Alcatel-Lucent, Holmdel, New Jersey 07733, USA \\ *lman@fotonik.dtu.dk
}

\begin{abstract}
We investigate the applicability of temporal multiplexing using four-wave mixing Bragg scattering for quantum frequency conversion. Various pump shapes are considered and we find that a large selectivity is possible for all the pump shapes.
\end{abstract}

(C) 2013 Optical Society of America

OCIS codes: (190.4380) Nonlinear optics, four-wave mixing; (270.5585) Quantum information and processing.

In quantum communication systems it is paramount to be able to manipulate the temporal shape as well as the frequency of the states, without destroying their other quantum properties [1]. One application is to convert the temporally wide states emitted from quantum memories to temporally shorter wavepackets for efficient transmission over optical fibers [2]. The first proposal for quantum frequency conversion was based on three-wave mixing (TWM) in optical crystals [3]. Another solution is four-wave mixing (FWM) in the form of Bragg scattering (BS), where two strong pumps $\mathrm{p}$ and $\mathrm{q}$ interact with a signal $\mathrm{s}$ and an idler $\mathrm{r}$, such that $\omega_{\mathrm{p}}+\omega_{\mathrm{s}}=\omega_{\mathrm{r}}+\omega_{\mathrm{q}}$ [4]. Recently, there has been an increasing interest in using TWM for temporal multiplexing [5], and in this paper we look at the applicability of FWM $\mathrm{BS}$ for this purpose. FWM by BS is described by the coupled mode equations

$$
\left(\partial_{z}+\beta_{\mathrm{r}} \partial_{t}\right) A_{\mathrm{r}}(z, t)=\mathrm{i} \gamma A_{\mathrm{q}}^{*}(z, t) A_{\mathrm{p}}(z, t) A_{\mathrm{s}}(z, t), \quad\left(\partial_{z}+\beta_{\mathrm{s}} \partial_{t}\right) A_{\mathrm{s}}(z, t)=\mathrm{i} \gamma A_{\mathrm{q}}(z, t) A_{\mathrm{p}}^{*}(z, t) A_{\mathrm{r}}(z, t),
$$

where $\partial_{z}$ and $\partial_{t}$ are the partial derivatives with respect to distance and time, respectively, $A_{\mathrm{p}}$ and $A_{\mathrm{q}}$ are the pumpshapes of pumps p and q, respectively, $A_{\mathrm{r}}$ and $A_{\mathrm{s}}$ are the shapes of the idler and signal, respectively, and $\gamma$ is the nonlinear coefficient, which is proportional to $\gamma_{\mathrm{K}}$, the Kerr nonlinearity. Finally $\beta_{\mathrm{r}}$ and $\beta_{\mathrm{s}}$ are the inverse group speeds of the idler and signal, respectively. The solutions are described by the Green function (GF) formalism

$$
A_{\mathrm{r}}(l, t)=\int_{-\infty}^{\infty} G_{\mathrm{rr}}\left(t ; t^{\prime}\right) A_{\mathrm{r}}\left(0, t^{\prime}\right) \mathrm{d} t^{\prime}+\int_{-\infty}^{\infty} G_{\mathrm{rs}}\left(t ; t^{\prime}\right) A_{\mathrm{s}}\left(0, t^{\prime}\right) \mathrm{d} t^{\prime}
$$

where we in this article assume that $A_{\mathrm{r}}\left(0, t^{\prime}\right)=0$ and we denote $t$ and $t^{\prime}$ the output and input times, respectively. Assuming that pump p co-propagates with the signal and pump q co-propagates with the idler one finds the solution [6]

$$
\begin{aligned}
& G_{\mathrm{rs}}\left(t ; t^{\prime}\right)=\mathrm{i} \bar{\gamma} A_{\mathrm{q}}^{*}\left(t-\beta_{\mathrm{r}} l\right) \mathrm{J}_{0}\left\{2 \bar{\gamma}\left[\xi\left(t, t^{\prime}\right) \eta\left(t, t^{\prime}\right)\right]^{1 / 2}\right\} A_{\mathrm{p}}\left(t^{\prime}\right) \mathrm{H}\left(t^{\prime}+\beta_{\mathrm{r}} l-t\right) \mathrm{H}\left(t-t^{\prime}-\beta_{\mathrm{s}} l\right), \\
& G_{\mathrm{sr}}\left(t ; t^{\prime}\right)=\mathrm{i} \bar{\gamma} A_{\mathrm{p}}^{*}\left(t-\beta_{\mathrm{s}} l\right) \mathrm{J}_{0}\left\{2 \bar{\gamma}\left[\xi\left(t, t^{\prime}\right) \eta\left(t, t^{\prime}\right)\right]^{1 / 2}\right\} A_{\mathrm{q}}\left(t^{\prime}\right) \mathrm{H}\left(t^{\prime}+\beta_{\mathrm{r}} l-t\right) \mathrm{H}\left(t-t^{\prime}-\beta_{\mathrm{s}} l\right),
\end{aligned}
$$

where $\bar{\gamma}=\gamma / \beta_{\mathrm{rs}}=\gamma /\left(\beta_{\mathrm{r}}-\beta_{\mathrm{s}}\right), l$ is the length of the fiber, $\mathrm{J}_{0}$ is the Bessel function of zeroth order, $\mathrm{H}$ is the Heaviside step function, and the normalized , distances" are defined as $\xi\left(t, t^{\prime}\right) \equiv \int_{t-\beta_{\mathrm{r}} l}^{t^{\prime}}\left|A_{\mathrm{q}}(s)\right|^{2} \mathrm{~d} s$ and $\eta\left(t, t^{\prime}\right) \equiv \int_{t^{\prime}}^{t-\beta_{\mathrm{s}} l}\left|A_{\mathrm{p}}(s)\right|^{2} \mathrm{~d} s$.

To better understand the properties of the Green function (GF) and to investigate its applicability as a tool for mode selectivity we introduce the singular value (Schmidt) decomposition [7] and the selectivity parameter $S$

$$
G_{\mathrm{rS}}\left(t ; t^{\prime}\right)=\sum_{n=0}^{\infty} v_{n}(t) \lambda_{n}^{1 / 2} u_{n}^{*}\left(t^{\prime}\right), \quad S \equiv \frac{\left|\lambda_{0}\right|^{2}}{\sum_{n=0}^{\infty}\left|\lambda_{n}\right|} \leq 1
$$

where $v_{n}$ and $u_{n}$ are the output and input modes, respectively, which comprise orthonormal sets individually, and $\lambda_{n}^{1 / 2}$ are the Schmidt coefficients, the squares of which are the mode conversion probabilities. One interesting limit is the case where the Schmidt decomposition only contains one term, in which case the GF is separable (free of temporal 


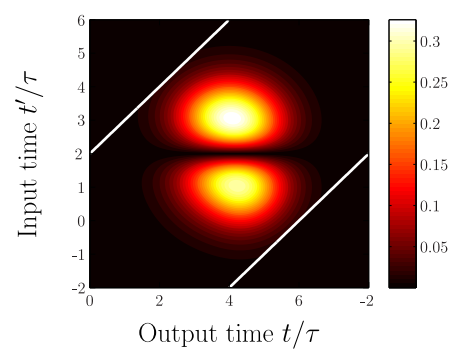

(a)

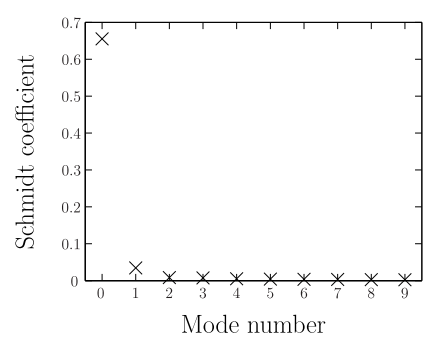

(b)

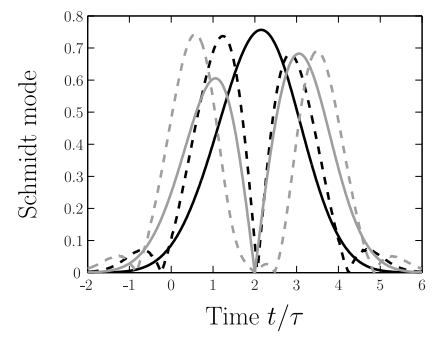

(c)

Fig. 1: Results for a Hermite-Gaussian pump of first order (p) and a zeroth order pump (q) with the fiber length $\beta l / \tau=4$ and $\bar{\gamma}=0.75$, where $l$ is the fiber length and $\tau$ is the width of the pumps. The absolute value of $G_{\mathrm{rs}}$ is shown in (a), (b) shows the first ten Schmidt coefficients, and (c) shows the first (solid) and the second (dashed) input (black) and output (gray) Schmidt modes.

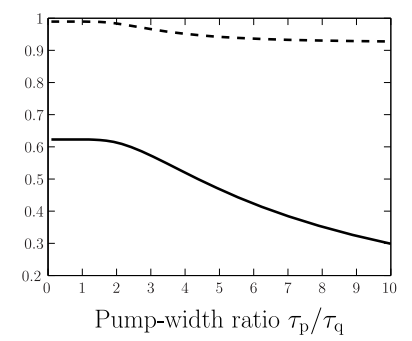

(a)

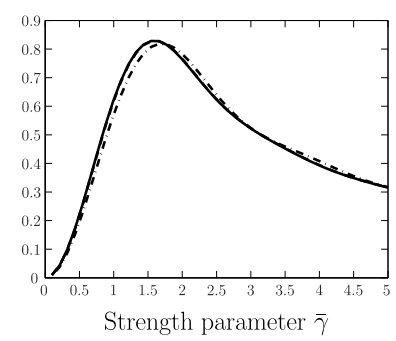

(b)

Fig. 2: (a) Selectivity (solid) and separability (dashed) as functions of the ratio of the two pump-widths $\tau_{\mathrm{p}} / \tau_{\mathrm{q}}$ for $\beta l / \tau_{\mathrm{q}}=4$ and $\bar{\gamma}=1$. (b) The selectivity as a function of the strength parameter for Hermite-Gaussian pumps of zeroth (solid), first (dashed), and second (dashed-dotted) order for $\beta l / \tau=4$.

entanglement). The degree of separability is described by the separability factor $S /\left|\lambda_{0}\right|$, and it is easily seen that the separability is unity for a separable GF. The advantage of the selectivity parameter is that it also describes the strength of the conversion of the fundamental mode, which is important for temporal mode selectivity.

The shapes of the GFs are rather complicated and in the rest of the article we study the GFs as well as their Schmidt decompositions numerically. First, we consider a Hermite-Gaussian pump of first order (p) and zeroth order (q) for the strength parameter $\bar{\gamma}=0.75$ and $\beta_{\mathrm{r}}=-\beta_{\mathrm{s}}=\beta$, see Fig. 1. In Fig. 1a the absolute value of $G_{\mathrm{rs}}$ is plotted, where the white lines bound the area where the step functions are nonzero, and with the Schmidt coefficients in Fig. $1 \mathrm{~b}$ which in this case gives us $S=0.43$. Finally, from Fig. 1c we see that with these pump choices the system accepts a Hermite-Gaussian of first-order but outputs a zeroth-order mode, in other words BS allows us to accept an arbitrary input signal and change the temporal shape of the idler as we see fit.

For temporal mode selectivity we are interested in as large a selectivity as possible, hence we now focus on the impact of various parameters on the selectivity. First we consider two Gaussian pumps with different widths, see Fig. 2a. From the figure it is seen that a high selectivity is obtainable for a wide range of pump widths, but that it falls off for large ratios of the pump widths. This is because the fiber length is fixed and the GF is „,cut-off” by the stepfunctions in the limit of large pump widths. Different pump shapes are also considered as a function of the strength parameter $\bar{\gamma}$, see Fig. 2b. For all the shapes there are optimal strength parameters, which are almost identical.

In this article we considered the possibility of using FWM BS for temporal mode selectivity. We found that for a variety of pump shapes and pump widths it was indeed possible to obtain a large selectivity factor.

\section{References}

1. S. Tanzilli et al., Nature (London) 437, 116-120 (2005).

2. C. Clausen et al., Nature 469, 508-511 (2011).

3. J. Huang and P. Kumar, Phys. Rev. Lett. 68, 2153-2156 (1992).

4. C. J. McKinstrie et al., Opt. Express 13, 9131-9142 (2005).

5. Y. Huang and P. Kumar, Opt. Lett. 38, 468-470 (2013).

6. C. J. McKinstrie et al., Phys. Rev. A 85, 053829 (2012).

7. H. J. McGuinness et al., Opt. Express 19, 17876-17907 (2011). 